\title{
Preoperative SARS-CoV-2 screening: Can it really rule out COVID-19?
}

\author{
Sylvain A. Lother, MD, FRCPC $\mathbb{D}$
}

Received: 26 May 2020 /Revised: 7 June 2020 / Accepted: 8 June 2020 /Published online: 23 June 2020

(C) Canadian Anesthesiologists' Society 2020

Expanded indications for testing the severe acute respiratory syndrome coronavirus 2 (SARS-CoV-2) have been recommended as health systems emerge from pandemic-related slowdowns. Routine preoperative screening prior to elective surgery has been broadly discussed. The putative benefits are intuitive: identify SARS-CoV-2 carriers before surgery to prevent adverse patient events, prevent further transmission, reduce consumption of resources and personal protective equipment, and improve hospital system efficiency. The harm associated with routine testing are less frequently considered. This editorial will address the deficiencies of real-time reverse transcriptase polymerase chain reaction (RT-PCR) as a method for detecting SARS-CoV-2 and provide information required to appropriately interpret test results.

The purpose of SARS-CoV-2 screening with RT-PCR is to detect viral genetic material (RNA) in the presymptomatic phase of infection. This incubation period for SARS-CoV-2 is protracted with initial low levels of viral RNA until replication increases in the hours or days leading up to symptom onset. ${ }^{1}$ On average, logarithmic viral replication and subsequent symptoms only start five to six days after exposure, but in some instances, this can be delayed up to 14 days. ${ }^{2}$ Before viral RNA reaches detectable thresholds, patients may appear well prior to elective surgery despite being exposed to SARS-CoV-2 in the preceding 14 days. If viral carriage is not detected by testing, patients may proceed with elective

S. A. Lother, MD, FRCPC ( $\square)$

Sections of Critical Care and Infectious Diseases, Department of Internal Medicine, University of Manitoba, JJ399- 820

Sherbrook St, Winnipeg, MB R3A 1R9, Canada

e-mail: slother@manitoba-physicians.ca surgery whereby signs and symptoms of coronavirus disease (COVID-19) may arise in the postoperative period, leading to adverse outcomes. ${ }^{3}$ While screening with RT-PCR may detect some presymptomatic preoperative patients, the window of diagnostic utility is small, and careful interpretation of negative and positive test results must be considered prior to altering the course of therapy.

\section{Screening in a low-risk population}

When evaluating any screening test, one must consider the sensitivity, specificity, and population prevalence of the target condition. Without a reference standard, measuring RT-PCR sensitivity for SARS-CoV-2 in asymptomatic patients remains an unresolved problem. ${ }^{4}$ As of 7 June 2020, clinical sensitivity has not been reported for any commercial tests in asymptomatic people. In the symptomatic cohort, considerable concern exists over false negative results, ranging from $11 \%$ to $40 \% .^{5}-{ }^{7}$ The probability of detecting SARS-CoV-2 also varies based on time from the exposure, being as low as $0 \%$ in the immediate days following exposure, $33 \%$ one day before symptom onset, $62 \%$ on the day of symptom onset, and peaking at $80 \%$ on day 3 of symptoms. ${ }^{8}$ Prevalence varies widely depending on the characteristics of the population of interest. Testing in the United Kingdom between 27 April and 10 May 2020 indicated that SARS-CoV-2 was present in $0.27 \%$ of the community population $(95 \%$ confidence interval, 0.17 to 0.41$).^{9}$ Thirty-three samples from 215 labouring women (15\%) in New York taken between 22 March and 4 April 2020 were positive, but only four of these women were symptomatic..$^{10}$ Highly variable 
testing accuracy and population characteristics will inevitably lead to false positives and false negatives.

To illustrate, consider an imaginary population of 1,000 patients awaiting elective surgery in whom the true population prevalence of SARS-CoV-2 is $2 \%$ (Table). Assume that all patients with SARS-CoV-2 are tested very soon before their symptoms emerge. The evidence cited above suggests a sensitivity of $70 \%$ and specificity of $95 \%$ are probably reasonable estimates. When the population prevalence is low the number of patients falsely diagnosed will be substantially greater than those correctly diagnosed. Some will be incorrectly cleared for surgery. How will health systems deal with these results?

\section{Interpreting a negative RT-PCR screening result for SARS-CoV-2}

Our imaginary population will largely test negative, so let's begin there. While the observed negative predictive value of $99 \%$ sounds attractive, health systems are given no guidance on how to manage these negative test results. Should these patients proceed to surgery? Can providers safely discontinue infection prevention and control measures? How should patients be counselled about their test results? What is their risk of postoperative COVID-19?

The answers to these questions are critically important prior to implementing widespread screening. While reports of RT-PCR sensitivity have varied widely, sensitivity is consistently low in the early or presymptomatic period. In our example, six patients with negative RT-PCR will be harbouring SARS-CoV-2 immediately before surgery. These patients are at high risk of morbidity and mortality. In a recent analysis of 278 patients undergoing elective surgery, the 30 -day mortality was $18.9 \%$ when SARS-CoV-2 infection was detected between seven days preoperatively and 30 days postoperatively. ${ }^{11}$ Without detection, patients may also propagate SARS-CoV-2 transmission. To prevent transmission to care providers and others, appropriate infection control measures should be maintained for any patients with possible exposures in the preceding 14 days, regardless of test results. ${ }^{12}$ This is supported by the American Society of Anesthesiologists/ Anesthesia Patient Safety Foundation joint statement, stating: "because false negatives may occur with testing, droplet precautions should be used by OR [operating room] staff for operative cases. Before performing AGMPs [aerosol-generating medical procedures], healthcare providers within the room should wear an N95 mask, eye protection, gown and gloves". ${ }^{13}$ In this context, it is unclear if testing will effectively minimize risk while reducing resource utilization and improving efficiency. Additionally, appropriate counselling on the benefits of proceeding with surgery weighted against the uncertain risks (based on false negative test results) in the postoperative period require careful discussion.

Interpretation of negative RT-PCR screening must be considered in the context of its many limitations. First, the probability of detecting viral RNA is extremely low in the first few days following exposure. ${ }^{8}$ If potential for a recent exposure exists, defer testing in favour of an observation period while limiting further exposure. ${ }^{14}$ Second, high-risk populations (e.g., close contact to a known COVID-19 case, recent travel, high-risk occupation) will increase the pre-test probability and the chance of false negative results. These individuals should isolate and limit exposures prior to proceeding to surgery. Lastly, rapid point of care tests are not yet validated in asymptomatic patients, so delays from sample collection to results (up to three days in some institutions) can lead to false reassurance. Patients may screen negative despite being in the early incubation phase. Viral load may increase in the time waiting for results. Proceeding to surgery without considering testing limitations could lead to devastating outcomes.

\section{Interpreting a positive RT-PCR screening result for SARS-CoV-2}

A positive RT-PCR result identifies a group of patients who may be infected with SARS-CoV-2 and should have elective surgeries delayed. This is clearly the most prudent path to take, and would allow for a period of observation, treatment, and recovery prior to pursuing elective surgery. Importantly, providers will need to be comfortable discussing the risks, benefits, and alternatives of delaying surgery further, and how their underlying comorbidities could affect COVID-19 outcomes.

The impact of false positive testing is unknown. Mass screening of patients in low prevalence settings will lead to considerable false positives, regardless of test specificity. Using our imaginary population, this test would yield a positive predictive value of only $22 \%$, leading to 49 false positive results for every 1,000 patients tested. These individuals are likely to experience further delays in elective procedures, unnecessary self-quarantine, and other psychosocial harms associated with receiving an erroneous COVID-19 diagnosis.

\section{Alternative strategy to routine SARS-CoV-2 screening before elective surgery}

Screening with RT-PCR is insensitive at detecting SARSCoV-2 early in its incubation phase. Alternate strategies are required to minimize harm to patients and providers. 
Perhaps the single best strategy to ensure patients arrive for surgery without subclinical SARS-CoV-2 infection is to mandate a period of strict self-quarantine for 14 days prior to elective surgery. Any prior exposures would have sufficient time to dissipate or enter the symptomatic phase. Patients who are asymptomatic after quarantine can safely proceed with surgery, without preoperative testing.

Nevertheless, not all patients will have the means to self-quarantine and this extreme strategy may increase social and economic stressors before elective surgery. Few health systems will have the resources to ensure patients self-isolate for this length of time. Thus, preoperative RTPCR testing should be considered one of a suite of tools in the multipronged approach to minimizing patient/provider risk as elective surgeries ramp up. The downsides of testing must be considered and incorporated into patient/provider discussions before proceeding to surgery and altering the clinical course.

In conclusion, SARS-CoV-2 preoperative screening programs are being considered to improve patient and provider safety, outcomes, and resource management. Nevertheless, the putative benefits of screening with RTPCR may not be realized because of low disease prevalence and poor test accuracy. Further evidence and guidance is needed to manage both false positive and false negative results. In addition to testing, alternative strategies that include self-isolation and other distancing measures are needed to enhance outcomes as we emerge from pandemic-related slowdowns.

\section{Dépistage préopératoire du SARS- CoV-2 : est-il véritablement possible d'exclure la présence de COVID-19?}

L'élargissement des indications pour les tests de dépistage du syndrome respiratoire aigu sévère du coronavirus 2 (SARS-CoV-2) a été recommandé alors que les systèmes de santé émergent des ralentissements liés à la pandémie. Le dépistage préopératoire de routine avant une chirurgie non urgente a été discuté dans les grandes lignes. Les avantages supposés d'un tel dépistage sont intuitifs : identifier les porteurs du SARS-CoV-2 avant leur chirurgie afin de prévenir les complications pour le patient, prévenir une transmission supplémentaire, réduire l'utilisation des ressources et des équipements de protection individuelle, et améliorer l'efficience du système hospitalier. Les effets nocifs d'un dépistage systématique sont moins souvent considérés. Cet éditorial abordera les écueils du test d'amplification par la polymérase avec transcription inverse en temps réel (RT-PCR) comme méthode de dépistage du SARS-CoV-2 et fournira les informations nécessaires à interpréter adéquatement les résultats de ce test.

L'objectif du dépistage du SARS-CoV-2 par RT-PCR est de détecter le matériel génétique viral (ARN) pendant la phase présymptomatique de l'infection. Cette période d'incubation du SARS-CoV-2 commence par de faibles taux initiaux d'ARN viral jusqu'à ce que la réplication augmente dans les heures ou jours précédant l'apparition des symptômes. ${ }^{1}$ En moyenne, la réplication virale logarithmique et les symptômes subséquents ne s'amorcent que cinq à six jours après l'exposition, et dans certains cas, jusqu'à 14 jours. ${ }^{2}$ Avant que l'ARN viral n'atteigne des seuils détectables, les patients pourraient apparaître en bonne santé avant une chirurgie non urgente malgré avoir été exposés au SARS-CoV-2 au cours des 14 jours précédents. Si la présence virale n'est pas dépistée par un test, les patients peuvent aller de l'avant avec leur chirurgie non urgente, à la suite de laquelle les signes et symptômes d'une atteinte au coronavirus (COVID-19) pourraient survenir en période postopératoire, entraînant des devenirs défavorables. ${ }^{3}$ Bien que le dépistage par RT-PCR puisse identifier certains patients préopératoires présymptomatiques, sa fenêtre d'utilité diagnostique est étroite, et une interprétation prudente des résultats négatifs et positifs du test doit être envisagée avant de modifier le déroulement du traitement.

\section{Dépistage dans une population à faible risque}

Lors de l'évaluation de tout test de dépistage, il faut tenir compte de sa sensibilité, de sa spécificité et de la prévalence dans la population de la condition ciblée. Sans norme de référence, la mesure de la sensibilité du RT-PCR pour dépister le SARS-CoV-2 chez les patients asymptomatiques demeure un problème non résolu. ${ }^{4}$ En date du 7 juin 2020, aucune sensibilité clinique n'a été rapportée pour un test commercial réalisé chez des personnes asymptomatiques. Dans la cohorte symptomatique, il existe d'importantes inquiétudes quant aux résultats faux négatifs, allant de $11 \%$ à $40 \% .^{5}-^{7} \mathrm{La}$ probabilité de dépistage du SARS-CoV-2 varie également en fonction du temps écoulé depuis l'exposition, étant aussi faible que $0 \%$ dans les jours suivant immédiatement l'exposition, $33 \%$ un jour avant l'apparition des symptômes, 62 \% le jour de l'apparition des symptômes, et atteignant un pic à $80 \%$ au troisième jour de présence de symptômes. ${ }^{8}$ La prévalence varie beaucoup en fonction des caractéristiques de la population à l'étude. Les tests réalisés au Royaume-Uni entre le 27 avril et le 10 mai 2020 ont indiqué que le SARS-CoV-2 était présent dans $0,27 \%$ de la 
Table Diagnostic accuracy of preoperative SARS-CoV-2 testing in a population with $2 \%$ disease prevalence

\begin{tabular}{llll}
\hline & SARS-CoV-2 & Healthy & Totals \\
\hline RT-PCR positive & $14(\mathrm{TP})$ & $49(\mathrm{FP})$ & 63 \\
RT-PCR negative & $6(\mathrm{FN})$ & $931(\mathrm{TN})$ & 937 \\
Totals & 20 & 980 & 1000 \\
\hline
\end{tabular}

Sensitivity $=14 /(14+6)=0.70$. Specificity $=931 /(931+49)=0.95$

Positive predictive value $=14 /(14+49)=0.22$

Negative predictive value $=931 /(931+6)=0.99$

$\mathrm{FN}$ = false negative; FP = false positive; RT-PCR = reverse transcriptase polymerase chain reaction; SARS-CoV-2 = severe acute respiratory syndrome coronavirus $2 ; \mathrm{TN}=$ true negative $\mathrm{TP}=$ true positive

population (intervalle de confiance $95 \%, 0,17$ à 0,41$){ }^{9}$ Trente-trois échantillons provenant de 215 femmes en travail obstétrical $(15 \%)$ à New York, prélevés entre le 22 mars et le 4 avril 2020, étaient positifs, mais seules quatre de ces femmes étaient symptomatiques. ${ }^{10} \mathrm{La}$ précision extrêmement variable du test et les caractéristiques démographiques entraîneront inévitablement des faux positifs et des faux négatifs.

À titre d'exemple, imaginons une population fictive de 1000 patients devant subir une chirurgie non urgente et dans laquelle la véritable prévalence du SARS-CoV-2 est de $2 \%$ (voir tableau). Partons du principe que tous les patients atteints par le SARS-CoV-2 sont testés peu après l'apparition de leurs symptômes. Selon les données probantes citées ci-dessus, une sensibilité de $70 \%$ et une spécificité de $95 \%$ seraient probablement des estimations raisonnables. Si la prévalence dans la population est faible, le nombre de patients diagnostiqués à tort sera considérablement plus élevé que le nombre de patients correctement diagnostiqués. Certains patients recevront à tort l'autorisation de subir leur chirurgie. Comment les systèmes de santé gèreront-ils ces résultats?

\section{Interprétation d'un résultat négatif de dépistage du SARS-CoV-2 par RT-PCR}

Prenons comme point de départ les résultats de test de notre population fictive, qui seront en grande partie négatifs. Alors que la valeur prédictive négative observée de $99 \%$ semble séduisante, les systèmes de santé n'ont reçu aucune directive sur la façon de gérer ces résultats de test négatifs. Ces patients devraient-ils subir leur chirurgie? Le personnel médical peut-il en toute sécurité ne prendre aucune mesure de prévention et de contrôle des infections? Comment faut-il conseiller les patients concernant les résultats de leur test? Quel est leur risque de COVID-19 postopératoire?
Avant d'instaurer un dépistage généralisé, il est crucial de répondre à ces questions. Alors que les comptes rendus portant sur la sensibilité du RT-PCR varient considérablement, la sensibilité est invariablement basse au début ou durant la période présymptomatique. Dans notre cohorte fictive, six patients dont le test RT-PCR était négatif seront porteurs du SARS-CoV-2 immédiatement avant leur chirurgie. Ces patients courent un risque élevé de morbidité et de mortalité. Dans une analyse récente de 278 patients subissant une chirurgie non urgente, la mortalité à 30 jours était de 18,9\% lorsqu'une infection au SARS-CoV-2 était décelée entre sept jours préopératoires et 30 jours postopératoires. ${ }^{11}$ Sans détection, ces patients pourraient également devenir des vecteurs de transmission du SARS-CoV-2. Afin de prévenir la transmission au personnel soignant et aux autres, il est important de maintenir des mesures de contrôle des infections adaptées pour tout patient ayant été possiblement exposé au virus dans les 14 jours précédents sa chirurgie, indépendamment des résultats de leur test. ${ }^{12}$ La déclaration conjointe de l'American Society of Anesthesiologists et de l'Anesthesia Patient Safety Foundation soutient cette approche : «Parce qu'il peut y avoir des faux négatifs dans les tests, les précautions contre les gouttelettes devraient être employées par le personnel de SOP [salle d'opération] pour les cas opératoires. Avant de réaliser une IMGA [intervention médicale générant des aérosols], les fournisseurs de soins de santé dans la salle devraient porter un masque N95, une protection oculaire, une blouse et des gants $» .{ }^{13}$ Dans ce contexte, il est impossible de déterminer si le dépistage minimisera effectivement les risques tout en réduisant l'utilisation des ressources et en améliorant l'efficacité. En outre, une consultation adaptée soupesant les avantages de la réalisation de la chirurgie et les risques incertains (si l'on se fonde sur les résultats de test faux négatifs) en période postopératoire justifie une discussion prudente.

Dans l'interprétation d'un dépistage négatif par RT-PCR, il faut tenir compte des nombreuses limitations 
TABLEAU Précision diagnostique du dépistage préopératoire du SARS-CoV-2 dans une population avec une prévalence de $2 \%$ de la maladie

\begin{tabular}{llll}
\hline & SARS-CoV-2 & Sains & Total \\
\hline RT-PCR positif & $14(\mathrm{VP})$ & 49 (FP) & 63 \\
RT-PCR négatif & $6(\mathrm{FN})$ & $931(\mathrm{VN})$ & 937 \\
Total & 20 & 980 & 1000 \\
\hline
\end{tabular}

Sensibilité $=14 /(14+6)=0,70$. Spécificité $=931 /(931+49)=0,95$

Valeur prédictive positive $=14 /(14+49)=0,22$

Valeur prédictive négative $=931 /(931+6)=0,99$

FN = faux négatif; FP = faux positif; RT-PCR = amplification par polymérase avec transcription inverse en temps réel; $\mathrm{SARS}-\mathrm{CoV}-2=$ syndrome respiratoire aigu sévère du coronavirus $2 ; \mathrm{VN}=$ vrai négatif; $\mathrm{VP}=$ vrai positif

de ce test. En premier lieu, la probabilité de détecter un ARN viral est extrêmement faible dans les premiers jours suivant l'exposition. ${ }^{8} \mathrm{Si}$ le potentiel d'une exposition récente existe, il convient de reporter le dépistage au profit d'une période d'observation tout en limitant toute exposition supplémentaire. ${ }^{14}$ En deuxième lieu, les populations à risque élevé (par ex., les personnes ayant eu des contacts étroits avec un cas connu de COVID-19, ayant voyagé récemment, ou exerçant un emploi à risque élevé) feront augmenter la probabilité pré-test et la probabilité de résultats faux négatifs. Ces personnes devraient s'isoler et limiter leur exposition avant de procéder à la chirurgie. Dernièrement, les tests rapides au chevet ne sont pas encore validés chez les patients asymptomatiques, et des délais entre la collecte des échantillons et les résultats (allant jusqu'à trois jours dans certains établissements) pourraient donner une impression de fausse sécurité. Les patients pourraient avoir un résultat négatif tout en étant au début de la phase d'incubation. La charge virale pourrait augmenter dans l'intervalle d'attente des résultats. La décision de réaliser une chirurgie sans tenir compte des limites du dépistage pourrait avoir des conséquences dévastatrices.

\section{Interprétation d'un résultat positif au dépistage du SARS-CoV-2 par RT-PCR}

Un résultat positif au dépistage par RT-PCR identifie un groupe de patients qui pourraient être infectés au SARS-CoV-2 et dont les chirurgies non urgentes devraient être retardées. Il s'agit clairement de la voie à suivre la plus prudente, et permettrait de disposer d'une période d'observation, de traitement et de récupération avant de réaliser une chirurgie non urgente. Il est important que les fournisseurs de soins se sentent à l'aise de discuter des risques, avantages et alternatives à un report de la chirurgie, et de la façon dont les comorbidités sous-jacentes des patients pourraient affecter leurs pronostics de COVID-19.

L'impact des tests faux positifs est inconnu. Le dépistage généralisé des patients dans un contexte de faible prévalence entraînera un nombre considérable de faux positifs, peu importe la spécificité du test. Dans notre population fictive, ce test donnerait une valeur prédictive positive de seulement $22 \%$, menant à 49 résultats faux positifs par tranche de 1000 patients testés. Ces personnes verront probablement leurs interventions non urgentes repoussées à nouveau, et subiront un isolement volontaire inutile et d'autres effets psychosociaux nocifs associés à la réception d'un diagnostic erroné de COVID-19.

\section{Une stratégie alternative au dépistage systématique du SARS-CoV-2 avant une chirurgie non urgente}

Le dépistage par RT-PCR n'est pas suffisamment sensible pour détecter le SARS-CoV-2 au début de la phase d'incubation. Il nous faut donc d'autres stratégies pour minimiser les effets néfastes pour les patients et les fournisseurs de soins de santé. La meilleure stratégie pour garantir que les patients se présentent pour leur chirurgie sans infection subclinique au SARS-CoV-2 serait peut-être d'exiger une période d'isolement volontaire stricte de 14 jours avant toute chirurgie non urgente. Toute exposition passée aurait ainsi le temps nécessaire pour montrer une non-infection ou entrer en phase symptomatique. Les patients asymptomatiques après cette période de quarantaine pourraient alors subir leur chirurgie en toute sécurité, sans dépistage préopératoire.

Il faut toutefois garder à l'esprit que tous les patients n'auront pas les moyens de se mettre en isolement volontaire, et cette stratégie extrême pourrait augmenter les facteurs de stress sociaux et économiques avant une chirurgie non urgente. Peu nombreux sont les systèmes de santé qui disposeront des ressources nécessaires pour 
garantir que les patients se placent en isolement volontaire pour une si longue période. Dès lors, le dépistage préopératoire par RT-PCR devrait être considéré comme un outil parmi d'autres dans une approche multidimensionnelle visant à minimiser le risque pour les patients et les fournisseurs de soins au fur et à mesure que les chirurgies non urgentes reprennent. Les inconvénients du dépistage doivent être pris en compte et intégrés dans les discussions entre patients et professionnels de la santé avant d'initier la chirurgie et de modifier le parcours clinique.

En conclusion, des programmes de dépistage préopératoire du SARS-CoV-2 sont envisagés pour améliorer la sécurité des patients et des professionnels de la santé, les pronostics, et la gestion des ressources. Toutefois, les avantages supposés d'un dépistage par RT-PCR pourraient ne pas se concrétiser en raison d'une faible prévalence de la maladie et du manque de précision du test. Des données probantes et des directives supplémentaires sont nécessaires pour gérer tant les résultats faux positifs que faux négatifs. Outre le dépistage, des stratégies alternatives comprenant l'isolement volontaire ainsi que d'autres mesures de distanciation sont nécessaires pour améliorer les devenirs alors que nous émergeons du ralentissement lié à la pandémie.

Disclosures None.

Funding statement None.

Editorial responsibility This submission was handled by Dr. Gregory L. Bryson, Deputy Editor-in-Chief, Canadian Journal of Anesthesia.

Déclarations Aucune.

Déclaration de financement Aucune.

Responsabilité éditoriale Cet article a été traité par Dr Gregory L. Bryson, rédacteur en chef adjoint, Journal canadien d'anesthésie.

\section{References}

1. He X, Lau EH, Wu P, et al. Temporal dynamics in viral shedding and transmissibility of COVID-19. Nat Med 2020; 26: 672-5.

2. Linton NM, Kobayashi T, Yang $Y$, et al. Incubation period and other epidemiological characteristics of, 2019 novel coronavirus infections with right truncation: a statistical analysis of publicly available case data. J Clin Med 2020; . https://doi.org/10.3390/ jcm9020538.

3. Lei $S$, Jiang $F, S u W$, et al. Clinical characteristics and outcomes of patients undergoing surgeries during the incubation period of COVID-19 infection. EClinicalMedicine 2020; . https://doi.org/ 10.1016/j.eclinm.2020.100331.

4. Woloshin S, Patel N, Kesselheim AS. False negative tests for SARS-CoV-2 infection - challenges and implications. N Engl J Med 2020; . https://doi.org/10.1056/NEJMp2015897.

5. Zhao J, Yuan $Q$, Wang $H$, et al. Antibody responses to SARSCoV-2 in patients of novel coronavirus disease 2019. Clin Infect Dis 2020; . https://doi.org/10.1093/cid/ciaa344.

6. Arevalo-Rodriguez I, Buitrago-Garcia D, Simancas-Racines D, et al. False-negative results of initial RT-PCR assays for COVID19: a systematic review. medRxiv 2020; DOI: https://doi.org/ 10.1101/2020.04.16.20066787.

7. Yang $Y$, Yang $M$, Shen $C$, et al. Evaluating the accuracy of different respiratory specimens in the laboratory diagnosis and monitoring the viral shedding of $2019-\mathrm{nCoV}$ infections. medRxiv 2020; DOI: https://doi.org/10.1101/2020.02.11.20021493.

8. Kucirka LM, Lauer SA, Laeyendecker O, Boon D, Lessler J. Variation in false-negative rate of reverse transcriptase polymerase chain reaction-based SARS-CoV-2 tests by time since exposure. Ann Intern Med 2020; . https://doi.org/10.7326/ M20-1495.

9. Office for National Statistics. Coronavirus (COVID-19) Infection Survey pilot: England, 14 May 2020. 2020. Available from URL: https://www.ons.gov.uk/peoplepopulationandcommunity/ healthandsocialcare/conditionsanddiseases/ bulletins/coronaviruscovid19infectionsurveypilot/ england14may2020 (accessed June 2010).

10. Sutton D, Fuchs $K$, D'Alton M, Goffman D. Universal screening for SARS-CoV-2 in women admitted for delivery. N Engl J Med 2020; 382: 2163-4.

11. Nepogodiev D, Glasbey JC, Li E, et al. Mortality and pulmonary complications in patients undergoing surgery with perioperative SARS-CoV-2 infection: an international cohort study. Lancet 2020; . https://doi.org/10.1016/S0140-6736(20)31182-X.

12. Livingston EH. Surgery in a time of uncertainty: a need for universal respiratory precautions in the operating room. JAMA 2020; . https://doi.org/10.1001/jama.2020.7903.

13. American Society of Anesthesiologists; Anesthesia Patient Safety Foundation. The ASA and APSF Joint Statement on Perioperative Testing for the COVID-19 Virus. April 292020. Available from URL: https://www.asahq.org/about-asa/ newsroom/news-releases/2020/04/asa-and-apsf-joint-statementon-perioperative-testing-for-the-covid-19-virus (accessed June 2020).

14. Engelman DT, Lother S, George I, et al. Ramping up delivery of cardiac surgery during the COVID-19 pandemic: a guidance statement from The Society of Thoracic Surgeons COVID-19 Task Force. Ann Thorac Surg 2020; . https://doi.org/10.1016/j. athoracsur.2020.05.002.

Publisher's Note Springer Nature remains neutral with regard to jurisdictional claims in published maps and institutional affiliations. 